\title{
Future scope and trends of natural chemistry
}

D.K. Awasthi ${ }^{1}$, Ragini Dubey ${ }^{2}$ and J. Pandey ${ }^{2,3 *}$

${ }^{1}$ Department of Chemistry, Sri. J.N.P.G. College, Lucknow, U.P.

${ }^{2,3}$ Department of Chemistry, Amity University, Lucknow, U.P. India

*Corresponding Author: J. Pandey, Department of Chemistry, Amity University, Lucknow, U.P. India.

Received date: December 01, 2020; Accepted date: December 10, 2020; Published date: January 16,2021

Citation: D.K. Awasthi, Ragini Dubey and J. Pandey (2021) Future scope and trends of natural chemistry, J. Pharmaceutics and Pharmacology

Research 4(1); DOI:10.31579/2693-7247/024

Copyright: (C) 2021, J. Pandey, This is an open access article distributed under the Creative Commons Attribution License, which permits unrestricted use, distribution, and reproduction in any medium, provided the original work is properly cited.

\begin{abstract}
Green science is else called sustainable sciences. The structure is utilized of chemical compounds and techniques that diminish age of perilous i.e. hazardous compound substances. Green science applies corner to corner the lifecycle of a chemical compound, including its assembling, use, plan, and at last removal. Green science is extremely useful in avoidance of contamination at the atomic level, it gives creative scientific arrangements, and it lessens the negative effects of compound on human health and the environment. Green science's 12 standards (Prevent squander, Maximize particle economy, Plan less risky concoction amalgamation, Design more secure synthetic concoctions and items, Use more secure solvents what's more, response conditions and Increase vitality productivity and so on.). Green science assume significant job in pharmaceutical in creating innovatory medicate conveyance strategies which are not so much poisonous but rather more valuable, viable with least symptoms and could help a large number of patients.
\end{abstract}

Key words: green science, pharmaceutical, perilous, hazardous, atomic level, amalgamation

\section{Introduction}

The most significant part of Green Chemistry is the idea of plan. Configuration or design is an announcement of human aim and one can't do plan unintentionally. It incorporates curiosity, arranging and methodical origination. The Twelve Principles of Green Chemistry are "plan rules" to assist scientists with accomplishing the deliberate objective of sustainability. Green Chemistry is portrayed via cautious arranging of the synthesis of chemicals and sub-atomic structure to decrease unfriendly results [1-4]. Through legitimate structure one can accomplish cooperative energies - not simply exchange offs. The Green Chemistry approach endeavors to accomplish sustainability at the sub-atomic level. On account of this objective, it isn't astonishing it has been applied to all industry segments. From aerospace, car i.e. automobile, electronics or gadgets, cosmetics, vitality, family unit items, pharmaceutical, to horticulture, there are many instances of effective uses of grant winning, financially serious advancements. The idea of Green Chemistry has had this enormous effect because of the way that it goes past the examination research center in segregation and has contacted industry, training, environment, and the overall population $[5,6]$.

\section{Basic Framework of Green Chemistry}

The three central points of the Green Chemistry structure can be summed up as:

1. Green Chemistry plans over all phases of the chemical (substance) lifecycle.

2. Green Chemistry tries to plan the inalienable idea of the synthetic items and procedures to decrease their characteristic danger.
3. Green Chemistry fills in as a strong arrangement of standards or structure models.

The target of Green Chemistry, to diminish hazards over all the life-cycle stages, has been demonstrated to be financially gainful. Hazard is characterized as the capacity to make unfavorable result people or the earth. Inherent risk of a concoction substance or a chemical procedure can be intended to be limited at each degree of a procedure, regardless of whether it is harmfulness, physical dangers (e.g., blast, combustibility) or worldwide hazards, for example, stratospheric ozone exhaustion. Dangers dependent on these hazards may ascend from the nature of feedstock and crude materials that are utilized in the chemical changes just as the last items that are made [7-9]. Cautious design will diminish or dispose of inherent hazards inside synthetic compounds and procedures; a plan dependent on the integration of Twelve Principles as one durable set [10]

Green chemistry works on both perilous i.e. risk and danger i.e. hazardous factor. These peril can be minimized by the reduction of the hazards proceeding with the cost and then the potential of exposure which can then be maintained [11-14]

To calculate the risk linked to the hazards of a particular substance we use the formula:

Risk $=\mathrm{f}($ exposure $*$ hazard $)$

\section{Green chemistry works on the following:}

Assessment of the methods to design the safer chemicals:

- Mechanism of examination of activity 
- $\quad$ Structure action relationship

- Avoidance of harmful functional groups

- Minimizing the bioavailability

Assessment of the reaction types:

- Addition reactions

- Substitution reactions

- Elimination reactions

Assessment and the design of energy efficient processes:

- Minimizing the auxiliary substances.

- The finest way of waste disposal.

Applications of Green Chemistry

1) Chemicals from $\mathrm{C}_{6} \mathrm{H}_{12} \mathrm{O}_{6}$ (glucose): These chemical compounds are a series of chemicals which can be produced on a completely gigantic scale to fulfil the international markets. Glucose i.e. $\mathrm{C}_{6} \mathrm{H}_{12} \mathrm{O}_{6}$ is one of the alternative for the product chemicals. Biotechnological plans are being used to manage the production of the fragrant compounds, compounds which includes catechol, hydroquinone \& adipic acid, all those compounds which be able to be fatal and be synthetic. Benzene $\left(\mathrm{C}_{6} \mathrm{H}_{6}\right)$ is the starting material used for these matters, concerning the changes of benzene amid $\left(\mathrm{C}_{6} \mathrm{H}_{12} \mathrm{O}_{6}\right)$ glucose can help in lowering the use of various reagents with few toxic. Those synthesis which takes place in water in place of organic solvents is more useful and beneficial [15].

2) Polysaccharide Polymers: They are a basic gathering of compounds that incorporate far reaching packages. They have their perilous results. The enormous scope of compounds can be misused. Polysaccharide in light of the fact that the feedstock must be utilized as starting materials because of the way that it's far extra ecologically/ environmentally feedstock. Those are natural i.e. organic and have the advantage of being sustainable or feasible, instead of oil feedstock. On the contrary side these don't have any interminable poisonousness to condition and soundness of people. A couple of more reactions are:

\section{The Green chemical reactions:}

1. Production of the aromatic amines which are without halide: Conventional assembling of the aromatic amines is finished by treating benzene with chlorine with the assistance of nitrogen and afterward uprooting chlorine with a totally new group (nucleophilic substitution). In this procedure nitrobenzene along with aniline is warmed within the sight of tetramethyl ammonium hydroxides to form tetramethyl-ammonium salts. The procedure abstains from utilizing halogenations intermediates.

2. Homogeneous catalysis and the molecule economic framework: This specific system was given by Trost. The point of this work is to diminish the futile side-effects of molecules framed during process [15].

\section{a. As green reagents:}

3. Liquid oxidation reactor: It permits safe oxidation of natural synthetic chemicals with pure oxygen. It can make reaction happen at low temperature and is very helpful. Because of this the measure of vent gas has been diminished.

4. Complexes produced by the green oxidative transmissions: different oxidation techniques have terrible environmental effects. The defile (metal particle) can be diminished by the utilization of molecular oxygen as the main oxidant. Numerous ligands have been propelled that are solid towards oxidative disintegration in oxidizing situations. Due to this present it's miles feasible to synthesize the stable unreasonable oxidation realm transition metal complexes.

5. Non phosgene isocynate blend: polyurethanes are the most extreme essential polymers. Polyurethanes are commonly produced by utilizing phosgene. Anyway phosgene has hindrance of being exceedingly harmful gas. Its intense surrender factor is lethality. To avoid the harmful gas phosgene, a strategy is utilized where the precursors of poly-urethanes and their isocyanate are being synthesized.

b. Reaction conditions for green solvents:

6. Making solvents stable: For solvents which have high amount and huge pertinence, the limit with regards to their poor effect on human wellbeing and the earth might be extremely high. Different solvents are agreeable and difficult to deal with [16].

7. Manufacturing the drugs (Drugs which are oligonucleotide): Artificial oligonucleotides drugs are expanding class of drug particles with wide scope of recuperating property. By and by, production is finished by utilizing HL-30 ${ }^{\mathrm{TM}}$ (the polystyrene dot) at a portion of $90 \mathrm{mmol} / \mathrm{g}$. It has various restricting attributes:

* Non-biodegradable

* Non-renewable

* It contributes about $\sim 40 \%$ of crude materials charges

* It is a solitary flexibly crude material

\section{c. Products of green synthetics:}

8. Other techniques for planning nitrites: Different structures of the compounds that can be poisonous are contemplated and changes are done to decrease the poisonousness. The mechanism which causes intense poisonousness is believed to be because of expulsion of hydrogen cyanide from cyanohydrins, contingent upon the idea of the replacement at alpha carbon function might be diminished or improved.

9. Polyaspartic acids (donlar's): By methods for the use of bio rational procedures the soybean blister nematode can be overseen: Soybean cyst nematode growth is as yet a rural problem. Being a piece of an inter disciplinary endeavor to discover a bio objective solution for the issue, different glycinoeclepin A analogues (a common bring forth upgrade of the nematode) have been created and the was testing done. Number of the analogs were found to have inhibitory activity on bring forth i.e. hatching of the soybean cyst, nematode eggs. These eggs in the lady can keep moving for eleven to twelve days in the soil [17].

10. Oxidation reagent \& catalysis: historically, a significant number of the oxidative reagents and the catalysts have been included poisonous substances, for example, metals which are heavy. Since these kind of substances were frequently utilized in incredibly enormous volumes required to change over a great many pounds of the petrochemicals, there was a critical inheritance of these metals being discharged to the earth and having significant negative impact not only on human wellbeing but also environment. It very well may be changed by the utilization of the benign substances.

11. Non-covalent derivatization: utilization of chemicals is needy upon development and breaking of the covalent bond. Science occurring without making the bond, both physical and chemical properties are altered and execution measures are upgraded by use of dynamic complexation which takes into consideration the transitory i.e. temporary developments of adjusted 
substance structures, the properties of particles can be changed for the time of the important to complete a specific function without the entirety of the waste that would be produced if full derivatization is actualized.

12. Supramolecular science: Research is at present progressing in the zone of supramolecular science to create reactions which can continue in the solid state without the utilization of solvents. The cycloaddition of the trans-1, 2-bis (4-pyridyl) ethylene is coordinated by resorcinol in solid state. This particular solid state reaction continues within the sight of UV light in $100 \%$ yield.

13. The Biometric multifunctional reagents: while engineered catalysis and reagents usually have fixated on completing of one discrete change. Though the controls may incorporate initiation, conformational changes, or few real changes and derivatizations.

14. Combinatorial Green Science: it's the science of having the option to form enormous quantities of substance i.e. chemical compounds quickly from a more minor perspective utilizing reaction networks. For example, lead it have huge no of derivatives. This science has empowered huge no of substances to be made and their properties surveyed without the size of the impacts of waste removal.

15. Energy center: The natural impact of vitality i.e. energy use are significant yet have not been as noticeable and as immediate as a portion of the perils that have not been presented by materials utilized in assembling, use and removal of synthetics. The advantage of catalysis is sensational in photochemistry. There is a need to structure substances and materials that are compelling, effective and economical at the catch, stockpiling and transportation.

16. Proliferation of dissolvable less responses: one of the 'solvent options' that is being: it is one of the alternatives of solvent that is being created in green science is that of the solvent less chemical reaction framework. The conveying of assembling process in the solvent less condition uses some non-customary conditions. These aides being developed of the isolation of product, partition and decontamination that will be solvent less also so as to expand the advantages.

\section{References}

1. Sheldon RA. (2005) Green solvents for sustainable organic synthesis: State of the art. Green Chem., 7, 267.

2. Clark JH, Luque R, Matharu AS. (2012) Green Chemistry, Biofuels, and Biorefinery. Annual Review of Chemical and Biomolecular Engineering. 3:183-207.

3. Ahluwalia VK and Kidwai M. (2004) New Trends in Green Chemistry. Anamaya Publishers, New Delhi.

4. Anastas PT, Horvath I, (2007) IT Innovations and Green Chemistry, Chem. Rev. 107:2169.

5. Wardencki W, Curyo J, Namieoenik J. (2004) green chemistrycurrent and future issues.

6. K. Hajela, J. Pandey, A. Dwivedy, J. D. Dhar, S. Sarkhel, P. R. Maulik, D. Velumurugan, (1999) Resolution, Molecular Structure and Biological Activities of the d- \&l- enantiomers of potent antiimplatation agent, dl-2[2-piperidinoethoxy] phenyl-3-2H1 benzopyran. Bioorg. Med. Chem., 2083-2090.

7. J. Pandey, R. Pal, A. Dwivedi, K. Hajela, (2002) Synthesis of 2,4dinitrophenylhydrazone derivatives of subs.1,2-diphenyl enthanones as possible anti-estrogenic and antibreast cancer agents. Arzneimittel-Forsch. 52, 39-44.

8. J. Pandey, K. Hajela, A. Dwivedi, (2004) Synthesis and Biological Efficacy of Some New 6H-Dibenzo [b, d] pyran-6-one and 6,6- dimethyl dibenzopyrans as Estrogen Antagonists/Modulators, Bioorg. Med. Chem. 12, 2239-2242.

9. J. Pandey, K. Hajela, (2014) Synthesis and biological activities of some substituted $6 \mathrm{H}$-dibenzo [b,d] pyran-6-one and 6,6-dimethyl 6H-dibenzo [b, d] pyran derivatives, Global J. Sci. Front. 14(4), 13-24.

10. A. Kulshrestha, J. Pandey, (2019) Multifarious stage synthesis of uniquely Substituted chromeno derivatives of carboxy and amino pyrimidine, Rasayan J. Chem., 12(3), 1660-1667.

11. A. Kulshrestha, J. Pandey, (2019) Knoevenagel Condensation Shadowed by Michael Addition \& O-Alkylation of Resorcinol, Malononitrile and Benzadehyde to form Pyrrolidine Piperidine and Morpholine Substituted Unique Benzopyran Derivatives in Dry $\mathrm{K}_{2} \mathrm{CO}_{3}$, A. J. Chem. 31(7), 1470-1472.

12. N. Singh, J. Pandey, J. Anireddy, (2019) Synthesis, Characterization, Computational Analysis And Antimicrobial Assay Of Novel Naphthyloxy And Naphthylphenoxy Derivatives, Int. J. Scien. Tech. Res., 8 (10), 784-789.

13. R. Bhatnagar, J. Pandey, D. Panhekar, (2020) Design, synthesis and biological activities of new alkylated isatin-derivatives, Int. J. Sci. Tech. Res., 9 (1), 740-742.

14. N. Singh, J. Pandey, J. Anireddy, (2020) Synthesis of novel 1,2,3,4-tetrahydro-isoquinoline derivatives, Int. J. Sci. Tech. Res., 9 (2), 3117-3120.

15. S. Satpute, N. Polkam, R. Kant, J. Shree Anireddy, D. Panhekar, J. Pandey, (2020) Design, synthesis and evaluation of $4 \mathrm{H}-$ Chromene-4-one analogues as potential Anti-bacterial and Antifungal agents, Chem. Biol. Lett., 7(1), 27-40.

16. R. Rupanwal, N. Singh, S. Satpute, D. Panhekar, J. Pandey, (2017) Copper (II) Salt Catalyzed Coupling Strategy towards Synthesis of Substituted Dibenzopyranones, A. J. Chem, 29(8), 1803-1805.

17. Sheldon RA, C.R. Acad Sci. Paris, IIc, Chimie/Chemistry, 2000, 3:541-551.

18. Iwata T, Miki H, Fujita Y. (1991) Ullmann's Encyclopedia of Industrial Chemistry, Vol. A19, VCH, Weinheim, p. 347

19. Micell Technology, accessed Dec. 1999.

20. P.T Anastas and T.C. Williamson, (1998) Green Chemistry: Frontiers in Benign chemical Synthesis and Processes. Oxford University Press, Oxford.

21. J.A. Hall, L.D. Vuocolo, I.D. Suckling, C.P. Horwitz, R.M. Allison, L.J. Wright, and T. Collins; (1999) Proceeding of 53rd APPITA Annual Conference, Rotorua, New Zealand. April 19-22.

22. P. Tundo and P.T. Anastas, (1998) Green Chemistry: Challenging Perspectives, Oxford University Press, Oxford. 